\title{
Simulations with different lattice Dirac operators for valence and sea quarks
}

\author{
Oliver Bär* \\ Center for Theoretical Physics, Massachusetts Institute of Technology, Cambridge, Massachusetts 02139, USA \\ Gautam Rupak ${ }^{\dagger}$ \\ Lawrence Berkeley National Laboratory, Berkeley, California 94720, USA \\ Noam Shoresh ${ }^{*}$ \\ Department of Physics, Boston University, Boston, Massachusetts 02215, USA
}

(Received 12 November 2002; published 19 June 2003)

\begin{abstract}
We discuss simulations with different lattice Dirac operators for sea and valence quarks. A goal of such a "mixed" action approach is to probe deeper the chiral regime of QCD by enabling simulations with light valence quarks. This is achieved by using chiral fermions as valence quarks while computationally inexpensive fermions are used in the sea sector. Specifically, we consider Wilson sea quarks and Ginsparg-Wilson valence quarks. The local Symanzik action for this mixed theory is derived to $\mathcal{O}(a)$, and the appropriate low energy chiral effective Lagrangian is constructed, including the leading $\mathcal{O}(a)$ contributions. Using this Lagrangian one can calculate expressions for physical observables and determine the Gasser-Leutwyler coefficients by fitting them to the lattice data.
\end{abstract}

DOI: 10.1103/PhysRevD.67.114505

PACS number(s): 11.15.Ha, 12.38.Gc, 12.39.Fe

\section{INTRODUCTION}

In order to extract predictions of QCD from numerical methods with controlled systematic errors, a lattice formulation is required for which the sources of deviations from QCD are understood and are under control. A significant source of systematic errors for present day lattice simulations are the light quark masses. Even the most powerful computers today do not allow simulations with up- and down-quark masses as light as realized in nature. Instead one simulates with heavier quark masses and fits the analytic predictions obtained in chiral perturbation theory $(\chi \mathrm{PT})$ to the data. The free parameters in the fit are the low energy constants of $\chi \mathrm{PT}$ [1], and once they are determined an extrapolation to small quark masses is possible [2,3]. Still, to perform the chiral extrapolation the quark masses must be small enough so that $\chi \mathrm{PT}$ is applicable. In practice one would require that nextto-leading order (NLO) $\chi \mathrm{PT}$ describe the data reasonably well. ${ }^{1}$

The present lattice simulations do not meet this requirement $[4-6]$. The data do not show the characteristic curvature predicted by NLO $\chi$ PT. In fact the data show a rather linear behavior which either means that higher orders in the chiral expansion are not negligible or worse, one is not in the chiral regime at all (see Bernard's part in [4]). In either case, simulations with lighter quark masses are required in order to apply $\chi \mathrm{PT}$ with confidence.

Lattice simulations with light fermions, especially sea

\footnotetext{
*Email address: obaer@1ns.mit.edu

${ }^{\dagger}$ Email address: grupak@lbl.gov

‡Email address: shoresh@bu.edu

${ }^{1}$ At next-to-next-to-leading order (NNLO) many new unknown parameters enter the chiral Lagrangian, which greatly reduces the predictive power of $\chi$ PT.
}

quarks, are computationally demanding and the numerical cost increases substantially with decreasing quark masses. Realistically only the least expensive fermions, Wilson and Kogut-Susskind, can be used on sufficiently large and fine lattices. Lattice fermions with better chiral properties are still too expensive to be used as sea quarks, and this situation is not likely to change in the near future. It is nevertheless expected that the next generation of TFLOP machines will make it possible to generate a few sets of unquenched configurations with sea quarks light enough to be in the chiral regime.

To obtain more information from these configurations they should (and will) be analyzed with various different valence quark masses, i.e. by studying partially quenched (PQ) QCD. By including lattice measurements with lighter valence quarks it is possible to penetrate further the chiral regime of QCD. This leads to more data points and would allow more reliable fits of PQ $\chi \mathrm{PT}[7]$ to the lattice data [2]. The reach of such simulations, however, is limited. The cost of light valence quarks also increases with the decreasing mass, and can become prohibitively high for quark masses that are still not very small. This is particularly true for Wilson fermions because of the explicit chiral symmetry breaking by the Wilson term.

An interesting idea for probing the chiral regime is to use different lattice fermions for the valence and sea quarks. In particular, by choosing lattice fermions with good chiral properties for the valence quarks, the valence quark mass can be made much smaller than in ordinary PQ simulations. A central goal of this strategy is the same as of PQ QCD-to explore a larger portion of the chiral regime by extracting more data points from a given set of unquenched configurations (see Fig. 1). This should result in more reliable estimates for the low-energy constants of $\chi \mathrm{PT}$ at NLO, the Gasser-Leutwyler coefficients. Furthermore, one might expect to reduce the size of explicit chiral symmetry breaking 


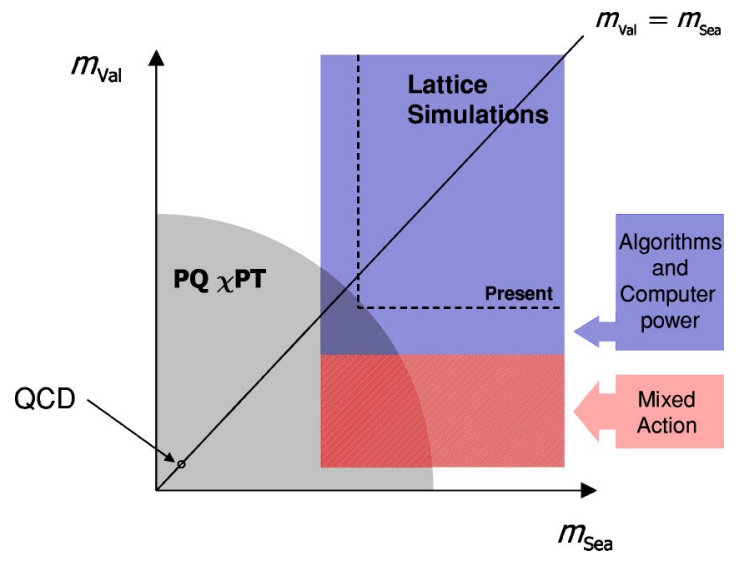

FIG. 1. Qualitative representation of the space of quark masses. The "chiral regime," where PQ $\chi$ PT can be applied, is the quartercircular region. The upper right rectangle, limited by the dashed line, describes the part of the space covered by present simulations. As current data suggest, there is very little or no overlap between that rectangle and the chiral regime. It is expected that improvement in algorithms and computer power will allow reducing the sea and valence quark masses in PQ simulations, as is represented by the enlargement of the previous rectangle. It is possible that the chiral region will be penetrated by such simulations, as shown by the small section of overlap between the enlarged rectangle and the chiral region. Finally, using chiral valence fermions in a mixed action simulation would make it possible to extend the reach of simulations significantly in the direction of lighter valence quarks.

by using Ginsparg-Wilson fermions at least for the valence quarks. This is a computationally affordable compromise of the lattice theorist's ideal of using Ginsparg-Wilson fermions for both valence and sea quarks.

In this paper we construct the low-energy chiral effective theory for a "mixed" lattice action, with explicit dependence on powers of the lattice spacing $a$ by first constructing the appropriate local Symanzik action. There are several reasons for taking this approach. First, the defining non-orthodox feature of the mixed action approach - the use of different Dirac operators for the sea and valence sectors-is purely a lattice artifact. This is a consequence of the fact that by construction all proper lattice fermions reproduce the same continuum physics, and therefore all mixed lattice theories reduce to PQ QCD in the continuum limit. An expansion in $a$ is thus a natural tool to investigate potential peculiarities of the mixed action formulation. Second, a theoretical understanding of the $a$ dependence in lattice simulations can guide the continuum limit, or allow the extraction of physical information directly from the lattice data, without taking the continuum limit first. Third, $\chi$ PT provides a useful framework for studying the chiral symmetry breaking due to the discrete space-time lattice. Effective theories of this type have been studied in several similar contexts [8-11].

What is dubbed here "mixed action methods" refers to a class of lattice theories corresponding to different choices of Dirac operators for the valence and sea quarks. In the next section we use a fairly simple example to illustrate the general framework of mixed lattice theories. We consider Wilson fermions for the sea quarks, together with valence fermions that satisfy the Ginsparg-Wilson relation. To describe the lattice action close to the continuum limit we construct the local Symanzik effective action up to $\mathcal{O}(a)$. The usual arguments used in the formulation of $\chi \mathrm{PT}$ are then applied to this effective action. This leads to a chiral expansion in which the dependence on the lattice spacing is explicit.

\section{THE CHIRAL EFFECTIVE ACTION}

\section{A. Lattice action}

In the following we always consider a hyper-cubic Euclidean space-time lattice with lattice spacing $a$. We assume either an infinite lattice or a finite lattice large enough that one can safely ignore finite volume effects.

The mixed lattice action that describes $N_{f}$ Wilson sea and $N_{V}$ Ginsparg-Wilson valence fermions has the structure

$$
S_{M}=S_{\mathrm{YM}}[U]+S_{W}\left[\bar{\psi}_{S}, \psi_{S}, U\right]+S_{\mathrm{GW}}\left[\bar{\psi}_{V}, \psi_{V}, U\right] .
$$

$U$ denotes the gauge field defined on the links of the lattice, $\psi_{S}\left(\bar{\psi}_{S}\right)$ are the sea quark (anti-quark) fields and $\psi_{V}\left(\bar{\psi}_{V}\right)$ denote vectors with $N_{V}$ anti-commuting valence quarks (antiquarks) and $N_{V} c$-number-valued ghost quarks (anti-quarks).

The precise choice for the gauge field action $S_{\mathrm{YM}}$ is irrelevant in the following, so we leave it unspecified. For the sea quarks we choose the Wilson action [12], given by

$$
\begin{gathered}
S_{W}=a^{4} \sum_{x} \bar{\psi}_{S}\left(D_{W}+m_{\mathrm{Sea}}\right) \psi_{S}(x), \\
\left.D_{W}=\frac{1}{2}\left\{\gamma_{\mu}\left(\nabla_{\mu}^{*}+\nabla_{\mu}\right)-\operatorname{ar} \nabla_{\mu}^{*} \nabla_{\mu}\right)\right\},
\end{gathered}
$$

where $m_{\text {Sea }}$ denotes the $N_{f} \times N_{f}$ quark mass matrix in the sea sector and $r$ the Wilson parameter. $\nabla_{\mu}^{*}, \nabla_{\mu}$ are the usual covariant, nearest neighbor backward and forward difference operators.

The action for the valence and ghost quarks is given by

$$
S_{G W}=a^{4} \sum_{x} \bar{\psi}_{V}\left\{D_{G W}+m_{\text {Val }}\left(1-\frac{1}{2} a D_{G W}\right)\right\} \psi_{V}(x) .
$$

The valence and ghost quark masses are contained in the $2 N_{V} \times 2 N_{V}$ mass matrix $m_{\text {Val }}$ of the form $m_{\text {Val }}$ $=\operatorname{diag}\left(M_{\mathrm{Val}}, M_{\mathrm{Val}}\right)$ where $M_{\mathrm{Val}}$ is an $N_{V} \times N_{V}$ matrix (i.e. each valence quark has a corresponding ghost field with the same mass). The Dirac operator $D_{G W}$ is assumed to be a local operator satisfying the Ginsparg-Wilson relation [13]

$$
\gamma_{5} D_{G W}+D_{G W} \gamma_{5}=a D_{G W} \gamma_{5} D_{G W} .
$$

Both the fixed-point Dirac operator and the overlap operator satisfy this relation [14-16]. For the following discussion, however, there is no need to specify $D_{G W}$ any further.

\section{B. Flavor symmetry of the lattice action}

When $m_{\mathrm{Sea}}=0, m_{\mathrm{Val}}=0$, and $r=0$, the flavor symmetry group of $S_{M}$ is 


$$
S U\left(N_{f}\right)_{L} \otimes S U\left(N_{f}\right)_{R} \otimes S U\left(N_{V} \mid N_{V}\right)_{L} \otimes S U\left(N_{V} \mid N_{V}\right)_{R}
$$

To see this it is convenient to write $S_{G W}$ and $S_{W}$ in terms of chiral components. The right- and left-handed sea quark fields are defined with the usual projectors $\frac{1}{2}\left(1 \pm \gamma_{5}\right)$. For the valence and ghost fields, one first defines the Hermitian operator

$$
\hat{\gamma}_{5}=\gamma_{5}\left(1-a D_{G W}\right),
$$

which is unitary as a consequence of Eq. (5). Valence rightand left-handed fields are now defined by [17]

$$
\begin{aligned}
& \bar{\psi}_{V, R}=\bar{\psi}_{V} \frac{1}{2}\left(1-\gamma_{5}\right), \quad \psi_{V, R}=\frac{1}{2}\left(1+\hat{\gamma}_{5}\right) \psi_{V}, \\
& \bar{\psi}_{V, L}=\bar{\psi}_{V} \frac{1}{2}\left(1+\gamma_{5}\right), \quad \psi_{V, L}=\frac{1}{2}\left(1-\hat{\gamma}_{5}\right) \psi_{V} .
\end{aligned}
$$

The fermionic actions can now be rewritten as

$$
\begin{aligned}
S_{W}= & a^{4} \sum_{x} \bar{\psi}_{S, L} \frac{1}{2} \gamma_{\mu}\left(\nabla_{\mu}+\nabla_{\mu}^{*}\right) \psi_{S, L}(x)+\bar{\psi}_{S, R} \frac{1}{2} \gamma_{\mu}\left(\nabla_{\mu}\right. \\
& \left.+\nabla_{\mu}^{*}\right) \psi_{S, R}(x)+\bar{\psi}_{S, L}\left(m_{\mathrm{Sea}}-\frac{1}{2} \operatorname{ar} \nabla_{\mu} \nabla_{\mu}^{*}\right) \psi_{S, R}(x) \\
& +\bar{\psi}_{S, R}\left(m_{\mathrm{Sea}}^{\dagger}-\frac{1}{2} \operatorname{ar}^{\dagger} \nabla_{\mu} \nabla_{\mu}^{*}\right) \psi_{S, L}(x),
\end{aligned}
$$

and

$$
\begin{aligned}
S_{G W}= & a^{4} \sum_{x} \bar{\psi}_{V, L} D_{G W} \psi_{V, L}(x)+\bar{\psi}_{V, R} D_{G W} \psi_{V, R}(x) \\
& +\bar{\psi}_{V, L} m_{\mathrm{Val}} \psi_{V, R}(x)+\bar{\psi}_{V, R} m_{\mathrm{Val}}^{\dagger} \psi_{V, L}(x) .
\end{aligned}
$$

Here, for reasons that will become clearer shortly, we consider $m_{\text {Sea }}, m_{\text {Val }}$ and $r$ to be matrices in flavor space, and identify the parameters that appear between right-handed anti-quarks and left-handed quarks as their Hermitian conjugates.

Clearly, when $m_{\mathrm{Sea}}=m_{\mathrm{Val}}=r=0$, Eqs. (10) and (11) are invariant under independent global rotations of the lefthanded and right-handed components of all quark fields:

$$
\psi_{X, \chi} \rightarrow g_{X, \chi} \psi_{X, \chi}, \quad \bar{\psi}_{X, \chi} \rightarrow \bar{\psi}_{X, \chi} g_{X, \chi}^{\dagger}, \quad X=S, V, \quad \chi=L, R,
$$

where $g_{S, L}$ and $g_{S, R}$ are in $S U\left(N_{f}\right)$, while $g_{V, L}$ and $g_{V, R}$ are in $S U\left(N_{V} \mid N_{V}\right)$. We conclude that flavor transformations belonging to the group in Eq. (6) are symmetries of the action Eq. (1) broken by the mass terms $m_{V a l}$ and $m_{S e a}$ and the Wilson term $r$.

It is convenient to treat these symmetry breaking parameters as spurion fields, i.e. assuming the transformation rules

$$
\begin{gathered}
m_{\mathrm{Val}} \rightarrow g_{V, L} m_{\mathrm{Val}} g_{V, R}^{\dagger}, \quad m_{\mathrm{Val}}^{\dagger} \rightarrow g_{V, R} m_{\mathrm{Val}}^{\dagger} g_{V, L}^{\dagger}, \\
m_{\mathrm{Sea}} \rightarrow g_{S, L} m_{\mathrm{Sea}} g_{S, R}^{\dagger}, \quad m_{\mathrm{Sea}}^{\dagger} \rightarrow g_{S, R} m_{\mathrm{Sea}}^{\dagger} g_{S, L}^{\dagger}, \\
r \rightarrow g_{S, L} r g_{S, R}^{\dagger}, \quad r^{\dagger} \rightarrow g_{S, R} r^{\dagger} g_{S, L}^{\dagger} .
\end{gathered}
$$

The mixed action $S_{M}$, even with non-vanishing mass and Wilson terms, is invariant under the combined transformations Eqs. (12),(13).

To complete this part we note that for a transformation to be a symmetry of the theory it must also leave unchanged the integration measure in the functional integral. It is a simple matter to show that the measure for the sea Wilson fermions is invariant under the global rotations in Eq. (12). The situation for the valence quarks is not quite so simple because of the operator $\hat{\gamma}_{5}$ in the chiral variation. It turns out, however, as has been shown in Ref. [18], that the measure is indeed invariant under the symmetry transformations considered here-the flavor non-singlet transformations. The last statement can be extended to the full valence sector, including the ghost fields. ${ }^{2}$

\section{Symanzik action}

We construct Symanzik's local effective theory which, close to the continuum, describes the same long-range physics as the discrete lattice action well below the momentum cutoff $1 / a[21-24]$.

Since the continuum action $S_{S}$ is designed to reproduce the same long-range correlation functions as the discrete lattice action $S_{M}$, it must have the same symmetries [Eq. (6)] as the underlying theory. Up to $\mathcal{O}(a)$, the quark operators that enter are of mass dimensions 3, 4, and 5, which include only quark bilinears. Moreover, the independent symmetry transformations acting separately on the sea and valence sectors requires that the quark bilinears do not mix the sectors. This implies that up to $\mathcal{O}(a)$, the fermionic operators in $S_{S}$ (as in $S_{M}$ ) are of two types - one built of sea quarks only and one of valence quarks.

It is straightforward to write down the $\mathcal{O}(a)$ Symanzik action $S_{S}$ using previous results concerning Wilson fermions $[25,26]$ and Ginsparg-Wilson fermions [17]. The details of the analysis are deferred to Appendix A-here we only quote the result (for the fermionic part of the action):

$$
\begin{aligned}
S_{S}= & \int d^{4} x\left[\bar{\psi}_{V}\left(D+\tilde{m}_{\mathrm{Val}}\right) \psi_{V}+\bar{\psi}_{S}\left(D+\tilde{m}_{\mathrm{Sea}}\right) \psi_{S}\right. \\
& \left.+a c_{S W} \bar{\psi}_{S} \sigma_{\mu \nu} F_{\mu \nu} \psi_{S}\right]+\mathcal{O}\left(a^{2}\right) .
\end{aligned}
$$

\footnotetext{
${ }^{2}$ The symmetry group that we write here is not the true symmetry group of the quantized theory. As discussed in $[19,20]$, the presence of ghost fields in the functional integral leads to constraints on the allowed symmetry transformations. However, for the derivation of the correct chiral Lagrangian it is possible to use the symmetry group in Eq. (6) [20].
} 
$\tilde{m}_{\text {Val }}$ and $\tilde{m}_{\text {Sea }}$ are renormalized masses. Two consequences of the exact chiral symmetry of the massless GinspargWilson fermions are: (a) there is no Pauli term $\bar{\psi} \sigma_{\mu \nu} F_{\mu \nu} \psi$ for the valence sector, and (b) the valence quark mass is only multiplicatively renormalized. No symmetry protects the Wilson sea quarks from getting an additive correction of the order of the cutoff $1 / a$.

It is useful at this point to collect the quark fields in a single quark field vector $\Psi$, and rewrite Eq. (14) as

$$
\begin{gathered}
S_{S}=\int \bar{\Psi}(D+\mathbf{m}) \Psi+\bar{\Psi} a \mathbf{c}_{\mathrm{SW}} \sigma_{\mu \nu} F_{\mu \nu} \Psi+\mathcal{O}\left(a^{2}\right), \\
\Psi=\left(\begin{array}{c}
\psi_{S} \\
\psi_{V}
\end{array}\right), \quad \mathbf{m}=\left(\begin{array}{cc}
\tilde{m}_{\mathrm{Sea}} & \\
& \tilde{m}_{\mathrm{Val}}
\end{array}\right), \quad a \mathbf{c}_{\mathrm{SW}}=\left(\begin{array}{lll}
a c_{S W} & \\
& 0
\end{array}\right) .
\end{gathered}
$$

\section{Symmetries of $S_{S}$ and $\chi$ PT for the mixed action}

We now turn to the construction of a low-energy effective theory for the "underlying" Symanzik action in Eq. (15). The method is completely analogous to the construction of the chiral Lagrangian in QCD [27]. ${ }^{3}$ The idea is that the spontaneous breaking of the approximate chiral symmetry gives rise to light pseudo Goldstone bosons, the light mesons, which at low energies are the only relevant degrees of freedom. The effective action is written in terms of local interactions of the pseudo-Goldstone fields. Since the pseudo-Goldstone bosons interact weakly at low energies, the action can be organized in a perturbative series.

All observables calculated are expanded in two small parameters,

$$
\epsilon \sim \frac{p^{2}}{\Lambda_{\chi}^{2}} \sim \frac{\hat{m}}{\Lambda_{\chi}^{2}} \text { and } \delta \sim \frac{\hat{a}}{\Lambda_{\chi}^{2}}
$$

where $p$ is the light meson momentum, $\Lambda_{\chi} \sim 1 \mathrm{GeV}$ is the chiral symmetry breaking scale, and $\hat{m}$ and $\hat{a}$ stand for matrix elements of ${ }^{4}$

$$
\hat{\mathbf{m}} \equiv 2 B_{0} \mathbf{m}, \quad \hat{\mathbf{a}} \equiv 2 W_{0} a \mathbf{c}_{\mathrm{SW}}
$$

\footnotetext{
${ }^{3}$ It should be noted that as of yet the construction of PQ $\chi$ PT from PQ QCD is not as well justified as the standard derivation of $\chi \mathrm{PT}$ from QCD. The arguments of the latter cannot be trivially extended to PQ QCD because they rely, in part, on the existence of a Hilbert space of physical states with a positive definite norm, which is absent in the presence of ghost fields. The same is also true for the mixed action. The validity of the $\chi \mathrm{PT}$ for the mixed action is thus on the same footing as that of PQ $\chi \mathrm{PT}$, which has been discussed in [20].

${ }^{4}$ Though the notation might obscure this fact, $\hat{\mathbf{m}}$ and $\hat{\mathbf{a}}$ both have mass dimension 2-they are the leading contributions to the squared mass of the pseudo Goldstone boson. We nevertheless use this notation as it makes the dependence on the quark masses and the lattice spacing more transparent.
}

$B_{0}$ and $W_{0}$ are dimensionful low-energy constants that appear in the effective theory at leading order [see Eq. (B1)]. They depend only on the high-energy scale $\Lambda_{\chi}$, and dimensional analysis reveals that the perturbative expansion is in fact in $m_{q} / \Lambda_{\chi}$ and $a \Lambda_{\chi}$. We follow Ref. [9] and choose the $\{\epsilon, \delta\}$ terms as leading order (LO) and the $\left\{\epsilon^{2}, \epsilon \delta\right\}$ terms as NLO in the effective Lagrangian, dropping $\mathcal{O}\left(a^{2}\right)$ contributions. The underlying hierarchy consistent with this ordering is $\{\epsilon, \delta\} \gg\left\{\epsilon^{2}, \epsilon \delta\right\} \gg \delta^{2}$, and the last inequality also implies $\epsilon \gg \delta$. This ordering is chosen for convenience and is somewhat arbitrary. In practice, the double expansion should be organized according to the actual relative sizes of the quark masses and the lattice spacing.

The effective Lagrangian is constructed from all operators that respect the symmetries of the underlying action $S_{S}$. The compact notation of Eq. (15) makes it easy to see that the symmetry group of $S_{S}$ to $\mathcal{O}(a)$ is

$$
S U\left(N_{f}+N_{V} \mid N_{V}\right)_{L} \otimes S U\left(N_{f}+N_{V} \mid N_{V}\right)_{R} .
$$

This symmetry group (treating $\hat{\mathbf{m}}$ and $\hat{\mathbf{a}}$ as spurion fields) is the same as that of PQ QCD with Wilson fermions. Indeed the effective action $S_{S}$ in Eq. (15) is the same as the effective Symanzik action for the PQ QCD Wilson action of Ref. [9], with a specific choice of $a \mathbf{c}_{\mathrm{SW}}$ that has support only in the sea sector. This fortunate similarity between the mixed action theory and the PQ QCD Wilson theory implies that the mixed low-energy chiral effective action has the same structure, at $\mathcal{O}(a)$, as the action of Wilson $\chi \mathrm{PT}$, introduced in Ref. [9], with the restriction that a $\mathbf{c}_{\mathrm{SW}}$ vanishes in the valence-ghost sector. The chiral Lagrangian to $\mathcal{O}(a)$, which describes both $\mathrm{W} \chi \mathrm{PT}$ and the mixed effective theory, is provided in Appen$\operatorname{dix} \mathrm{B}$.

\section{E. Application: Meson mass}

In this subsection we give an example for the use of the mixed chiral Lagrangian. For simplicity we take all the sea quarks and all the valence quarks to be (separately) degenerate, and the Wilson parameter to be a flavor singlet in the sea sector. This amounts to setting

$$
\hat{\mathbf{m}}=\operatorname{diag}\left(\hat{m}_{\text {Sea }}, \hat{m}_{\text {Val }}\right), \quad \hat{\mathbf{a}}=\operatorname{diag}(\hat{a}, 0)
$$

The number of sea quark flavors is taken to be $N_{f}=3$. We consider the expression for the mass of the flavor charged meson with valence quark flavor indices $A B(A \neq B)$ to NLO. Using the relation between the mixed chiral effective theory and $\mathrm{W} \chi \mathrm{PT}$, one can obtain the result straightforwardly by taking the mass formula from Ref. [9] with the values for $\hat{\mathbf{m}}$ and $\hat{\mathbf{a}}$ given by Eq. (20). We find

$$
\begin{aligned}
\left(M_{\mathrm{AB}}^{2}\right)_{\mathrm{NLO}}= & \hat{m}_{\mathrm{Val}}+\frac{\hat{m}_{\mathrm{Val}}}{48 f^{2} \pi^{2}}\left[\hat{m}_{\mathrm{Val}}-\hat{m}_{\mathrm{Sea}}-\hat{a}+\left(2 \hat{m}_{\mathrm{Val}}-\hat{m}_{\mathrm{Sea}}\right.\right. \\
& \left.-\hat{a}) \ln \left(\hat{m}_{\mathrm{Val}}\right)\right]-\frac{8 \hat{m}_{\mathrm{Val}}}{f^{2}}\left[\left(L_{5}-2 L_{8}\right) \hat{m}_{\mathrm{Val}}+3\left(L_{4}\right.\right. \\
& \left.\left.-2 L_{6}\right) \hat{m}_{\mathrm{Sea}}+3\left(W_{4}-W_{6}\right) \hat{a}\right] .
\end{aligned}
$$


Here, the parameters $L_{i}$ are the Gasser-Leutwyler coefficients, and $W_{4}$ and $W_{6}$ are additional low-energy constants that enter the chiral Lagrangian at NLO. Note that for $\hat{a}=0$ the expression for PQ $\chi \mathrm{PT}$ (calculated in $[2,28,29]$ ) is recovered.

Equation (21) demonstrates the analytic connection between QCD and the simulated mixed action theory. It shows the latter to be a calculation with controlled systematic errors. From fitting the equation to the appropriate data from numerical simulations one can obtain an estimate for the linear combinations of Gasser-Leutwyler coefficients that appear in it.

Examining Eq. (21) one can also appreciate the potential advantage of using a mixed lattice action. In simulations using Wilson fermions in both sea and valence sectors, an equation similar to Eq. (21) holds [see Eq. (B5)]. In that case, the range of valence quark masses that can be simulated might be too small to convincingly show the curvature coming from the quadratic dependence and the logarithms that enter at NLO. By using Ginsparg-Wilson fermions for the valence quarks one can vary the valence quark masses over a wider range. The expected NLO curvature, on which the extraction of the Gasser-Leutwyler coefficients depends, is consequently much more likely to be seen.

Finally, comparison with the result for $\mathrm{W} \chi \mathrm{PT}$, Eq. (B5), reveals that the latter depends on twice the number of $W_{i}$ coefficients. This is fortunate for the mixed theory as it makes the predictions of the effective theory less dependent on parameters that have no particular relevance to QCD.

To understand this simplification in the expression for the meson mass, consider the relation between the symmetries of the mixed action and those of PQ Wilson action. On the one hand the massless mixed theory has exact chiral symmetry in the valence sector, which the Wilson action does not. On the other hand, the valence and sea quarks of the Wilson action have the same type of Dirac operator which allows mixing between the sectors- a transformation which is not a symmetry of the mixed action formulation. At $\mathcal{O}(a)$, however, the breaking of the sea-valence symmetry in the mixed theory does not yet show up, and thus the simpler expressions arise due to the larger chiral symmetry.

\section{SUMMARY}

In this paper we discuss lattice simulations with different fermions for sea and valence quarks. As a particular example we have studied here the case with Wilson sea quarks and Ginsparg-Wilson valence quarks. Using Symanzik's effective action for lattice theories as an intermediate step, we have derived the form of the low-energy chiral Lagrangian for the mixed theory to $\mathcal{O}(a)$. The construction shows that simulations with the mixed action provide as controlled an approximation to QCD as partially quenched simulations. This is to be expected since the mixed action reduces to PQ QCD in the continuum limit.

The goal of the mixed action approach is similar to that of PQ QCD. The use of smaller valence quark masses allows one to probe deeper the chiral regime of QCD and obtain additional information on the low-energy constants, the
Gasser-Leutwyler coefficients. Furthermore, the use of chiral lattice fermions in the valence sector, instead of Wilson fermions, makes it possible to simulate much lighter valence quarks. This leads to more data points obtained on the lattice and consequently to more reliable fits of $\chi \mathrm{PT}$ to the data.

Here we have demonstrated the mixed action approach for Wilson sea quarks and Ginsparg-Wilson valence quarks taking into account the leading $\mathcal{O}(a)$ contributions. An important extension of this analysis is the inclusion of $\mathcal{O}\left(a^{2}\right)$ effects. First of all, the lattice spacing determined by the unquenched configurations is possibly not small enough to safely neglect the $\mathcal{O}\left(a^{2}\right)$ corrections. If one wants to fit the lattice data directly to equations like Eq. (21) without taking the continuum limit first, the $\mathcal{O}\left(a^{2}\right)$ corrections should be included to obtain better fits. Second, the $\mathcal{O}(a)$ effects are generated here only by the Wilson sea quarks. Many unquenched simulations are in fact performed with nonperturbatively $\mathcal{O}(a)$-improved Wilson fermions for the sea quarks. The leading corrections for these simulations are of $\mathcal{O}\left(a^{2}\right)$ and need to be computed in order to know how the continuum limit is approached.

While valuable, it should also be noted that the inclusion of $\mathcal{O}\left(a^{2}\right)$ effects in the chiral Lagrangian framework is likely to be a hard task. The main difficulty arises from the many new operators that enter the Symanzik action at this order. Some of these operators break Lorentz invariance, while several others break the chiral symmetry and require the introduction of additional spurion fields.

The approach proposed here should be also studied with other combinations for the lattice fermions. In particular, the case with staggered sea quarks is interesting, since staggered fermions are computationally cheaper. At present the lightest dynamical quark masses are achieved with staggered fermions. It is well known that applying staggered fermions to QCD involves a theoretical uncertainty and is possibly uncontrolled. Consequently, predictions from chiral perturbation theory for staggered fermions would also serve as a test of this discretization method [7].

Finally, the cost of simulations of a mixed action is roughly the sum of the cost of generating a set of unquenched gauge field configurations plus that of analyzing quenched simulations with Ginsparg-Wilson fermions. Thus, we can expect that in the near future simulations with a mixed action will become feasible.

\section{ACKNOWLEDGMENTS}

We acknowledge support in part by U.S. DOE grants DFFC02-94ER40818, DE-AC03-76SF00098, DE-FG0396ER40956/A006 and DE-FG02-91ER40676. We thank Maarten Golterman and Steve Sharpe for their comments on this manuscript. G.R. would like to thank the Department of Physics, Boston University and the Benasque Center for Science, Benasque, Spain for kind hospitality during part of this work.

\section{APPENDIX A: SYMANZIK ACTION FOR THE WILSON AND GINSPARG-WILSON ACTIONS}

In this appendix we derive Eq. (14) for the Symanzik action describing the mixed theory to $\mathcal{O}(a)$. As has been 
stated in the text, to this order the Symanzik action is simply the sum of the local effective actions for the valence and the sea sectors.

The local Symanzik action for Wilson fermions has been derived in $[25,26]$. One first lists all the operators of mass dimension no greater than 5 , which respect the symmetries of the Wilson lattice action (the appropriate power of $a$ is inserted to complete the dimensions of terms in the Lagrangian to 4). The operators of dimension 4 (which are $a$ independent) make up, by construction, the continuum action of QCD.

Because the Wilson term explicitly breaks the chiral symmetry, it is expected that the quark mass be additively renormalized, and the size of the correction should be of the order of the cutoff scale 1/a. Indeed, the only dimension 3 operator is $\bar{\psi} \psi$, which appears in the action with a coefficient proportional to $1 / a$ and has precisely this effect.

There are several operators of mass dimension 5 that are allowed by the symmetries. Some of these operators can be eliminated using the leading order equations of motion. Others have the same structure as the mass and kinetic operators that already appear in the QCD action, and have the effect of renormalizing the quark masses and the gauge coupling. Finally, a single term is left-the Pauli term: $\bar{\psi} \sigma_{\mu \nu} F_{\mu \nu} \psi$. Note that the Pauli term breaks the chiral symmetry, and is therefore allowed only because of the Wilson term. Putting it all together, the Symanzik action for the Wilson sea sector is

$$
\int d^{4} x\left[\bar{\psi}_{S}\left(D+\tilde{m}_{\mathrm{Sea}}\right) \psi_{S}+a c_{S W} \bar{\psi}_{S} \sigma_{\mu \nu} F_{\mu \nu} \psi_{S}\right]+\mathcal{O}\left(a^{2}\right),
$$

where $\tilde{m}_{\mathrm{Sea}}$ is the renormalized sea quark mass, and $c_{S W}$ is an unknown coefficient.

The analysis for the Ginsparg-Wilson valence quarks is similar. This may seem confusing due to the fact that some of the chiral projectors on the lattice are written in terms of $\hat{\gamma}_{5}$, and not $\gamma_{5}$ as in the continuum theory. However, it has been shown in Refs. [30,31], that the chiral symmetry of the Ginsparg-Wilson lattice action leads to exactly the same chiral Ward identities which appear in the continuum. Hence, by imposing the usual chiral symmetry on the Symanzik action, the effective theory correctly reproduces the consequences of the lattice chiral symmetry.
Due to the exact chiral symmetry the valence quark mass gets renormalized only multiplicatively and the Pauli term is absent. Consequently, after considering the renormalizations of gauge coupling and quark masses, the Symanzik action for the valence Ginsparg-Wilson quarks contains no $\mathcal{O}(a)$ part [the Ginsparg-Wilson lattice action is automatically $\mathcal{O}(a)$ improved [17]]:

$$
\int d^{4} x\left[\bar{\psi}_{V}\left(D+\tilde{m}_{\mathrm{Val}}\right) \psi_{V}\right]+\mathcal{O}\left(a^{2}\right)
$$

Equation (14) is the sum of Eq. (A1) and Eq. (A2).

\section{APPENDIX B: $\mathrm{W}_{\chi}$ PT RESULTS}

We present the $\mathrm{W} \chi \mathrm{PT}$ Lagrangian which also describes the mixed theory to $\mathcal{O}(a)$. Interested readers should consult Ref. [9] for further details on $\mathrm{W}_{\chi} \mathrm{PT}$. We also provide the expression for the mass of a flavor charged meson for comparison with the mixed theory result.

The $\mathrm{W} \chi \mathrm{PT}$ Lagrangian is constructed out of operators that respect all the symmetries of the underlying theory in Eq. (15), with explicit flavor axial symmetry breaking terms constructed out of $\hat{m}$ and $\hat{a}$. As described in the text, the LO Lagrangian is linear in $\epsilon$ and $\delta$ :

$$
\mathcal{L}_{2}=\frac{f^{2}}{4}\left\langle\partial \Sigma \partial \Sigma^{\dagger}\right\rangle-\frac{f^{2}}{4}\left\langle(\hat{m}+\hat{a}) \Sigma^{\dagger}+\Sigma\left(\hat{m}^{\dagger}+\hat{a}^{\dagger}\right)\right\rangle .
$$

Here the angled brackets stand for the super-trace over the flavor indices:

$$
\begin{aligned}
\langle\Gamma\rangle & =\operatorname{str}(\Gamma)=\sum_{i} \eta_{i} \Gamma_{i i}, \\
\eta_{i} & =\left\{\begin{array}{cc}
1, & i \text { is a quark flavor index, } \\
-1, & i \text { is a ghost flavor index, }
\end{array}\right.
\end{aligned}
$$

and $\Sigma=\exp (2 i \Pi / f)$ is a non-linear representation of the meson fields.

The NLO Lagrangian is ${ }^{5}$

$$
\begin{aligned}
\mathcal{L}_{4}= & -L_{1}\left\langle\partial \Sigma \partial \Sigma^{\dagger}\right\rangle^{2}-L_{2}\left\langle\partial_{\mu} \Sigma \partial_{\nu} \Sigma^{\dagger}\right\rangle\left\langle\partial_{\mu} \Sigma \partial_{\nu} \Sigma^{\dagger}\right\rangle-L_{3}\left\langle\left(\partial \Sigma \partial \Sigma^{\dagger}\right)^{2}\right\rangle+L_{4}\left\langle\partial \Sigma \partial \Sigma^{\dagger}\right\rangle\left\langle\hat{m} \Sigma^{\dagger}+\Sigma \hat{m}^{\dagger}\right\rangle+W_{4}\left\langle\partial \Sigma \partial \Sigma^{\dagger}\right\rangle\left\langle\hat{a} \Sigma^{\dagger}+\Sigma \hat{a}^{\dagger}\right\rangle \\
& +L_{5}\left\langle\partial \Sigma \partial \Sigma^{\dagger}\left(\hat{m} \Sigma^{\dagger}+\Sigma \hat{m}^{\dagger}\right)\right\rangle+W_{5}\left\langle\partial \Sigma \partial \Sigma^{\dagger}\left(\hat{a} \Sigma^{\dagger}+\Sigma \hat{a}^{\dagger}\right)\right\rangle-L_{6}\left\langle\hat{m} \Sigma^{\dagger}+\Sigma \hat{m}^{\dagger}\right\rangle^{2}-W_{6}\left\langle\hat{m} \Sigma^{\dagger}+\Sigma \hat{m}^{\dagger}\right\rangle\left\langle\hat{a} \Sigma^{\dagger}+\Sigma \hat{a}^{\dagger}\right\rangle \\
& -L_{7}\left\langle\hat{m} \Sigma^{\dagger}-\Sigma \hat{m}^{\dagger}\right\rangle^{2}-W_{7}\left\langle\hat{m} \Sigma^{\dagger}-\Sigma \hat{m}^{\dagger}\right\rangle\left\langle\hat{a} \Sigma^{\dagger}-\Sigma \hat{a}^{\dagger}\right\rangle-L_{8}\left\langle\hat{m} \Sigma^{\dagger} \hat{m} \Sigma^{\dagger}+\Sigma \hat{m}^{\dagger} \Sigma \hat{m}^{\dagger}\right\rangle-W_{8}\left\langle\hat{a} \Sigma^{\dagger} \hat{m} \Sigma^{\dagger}+\Sigma \hat{a}^{\dagger} \Sigma \hat{m}^{\dagger}\right\rangle .
\end{aligned}
$$

These Lagrangians describe both the mixed and the PQ Wilson lattice actions. In the mixed theory $\hat{a}$ has support only in the

\footnotetext{
${ }^{5}$ There has been an error in Ref. [9] in the sign of some of the terms in $\mathcal{L}_{4}$. The form that appears here is the correct one.
} 
sea-sea sector. We comment that Eqs. (B1), (B3) contain ordinary $\chi$ PT. Moreover, since the low-energy constants $L_{i}$ 's and $W_{i}$ 's are independent of $\hat{m}$ and $\hat{a}$ and this theory becomes the familiar $\chi \mathrm{PT}$ in the sea-sea sector when $a \rightarrow 0$, the $L_{i}$ 's are exactly the Gasser-Leutwyler coefficients of ordinary $\chi \mathrm{PT}$.

Next, we provide the $\mathrm{W} \chi \mathrm{PT}$ expression for the mass of the flavor charged meson defined in Sec. II E. We consider the case where

$$
\hat{\mathbf{m}}=\operatorname{diag}\left(\hat{m}_{\text {Sea }}, \hat{m}_{\text {Val }}\right), \quad \hat{\mathbf{a}}=\operatorname{diag}\left(\hat{a}_{\text {Sea }}, \hat{a}_{\text {Val }}\right)
$$

[compare with Eq. (20)]. One obtains

$$
\begin{aligned}
\left(M_{\mathrm{AB}}^{2}\right)_{\mathrm{NLO}}= & \left(\hat{m}_{\mathrm{Val}}+\hat{a}_{\mathrm{Val}}\right)+\frac{\left(\hat{m}_{\mathrm{Val}}+\hat{a}_{\mathrm{Val}}\right)}{48 f^{2} \pi^{2}}\left[\left(\hat{m}_{\mathrm{Val}}+\hat{a}_{\mathrm{Val}}\right)-\left(\hat{m}_{\mathrm{Sea}}+\hat{a}_{\mathrm{Sea}}\right)+\left(2\left(\hat{m}_{\mathrm{Val}}+\hat{a}_{\mathrm{Val}}\right)-\left(\hat{m}_{\mathrm{Sea}}+\hat{a}_{\mathrm{Sea}}\right)\right) \ln \left(\hat{m}_{\mathrm{Val}}+\hat{a}_{\mathrm{Val}}\right)\right] \\
& -\frac{8 \hat{m}_{\mathrm{Val}}}{f^{2}}\left[\left(L_{5}-2 L_{8}\right) \hat{m}_{\mathrm{Val}}+3\left(L_{4}-2 L_{6}\right) \hat{m}_{\mathrm{Sea}}+3\left(W_{4}-W_{6}\right) \hat{a}_{\mathrm{Sea}}\right]-\frac{8 \hat{a}_{\mathrm{Val}}}{f^{2}}\left[\left(L_{5}+W_{5}-2 W_{8}\right) \hat{m}_{\mathrm{Val}}+3\left(L_{4}\right.\right. \\
& \left.\left.-W_{6}\right) \hat{m}_{\mathrm{Sea}}\right] .
\end{aligned}
$$

To obtain the expression appropriate for common lattice simulations, in which the Wilson term is the same for all flavors, one sets $\hat{a}_{\mathrm{Val}}=\hat{a}_{\mathrm{Sea}}$ in the last equation. The meson mass for the mixed action [Eq. (21)] can be obtained by setting $\hat{a}_{\mathrm{Val}}=0$.

[1] J. Gasser and H. Leutwyler, Nucl. Phys. B250, 465 (1985).

[2] S.R. Sharpe and N. Shoresh, Phys. Rev. D 62, 094503 (2000).

[3] A.G. Cohen, D.B. Kaplan, and A.E. Nelson, J. High Energy Phys. 11, 027 (1999).

[4] C. Bernard et al., hep-lat/0209086.

[5] S. Dürr, hep-ph/0209319.

[6] qq+q, Collaboration, F. Farchioni, C. Gebert, I. Montvay, and L. Scorzato, hep-lat/0209142.

[7] C.W. Bernard and M.F.L. Golterman, Phys. Rev. D 49, 486 (1994).

[8] S.R. Sharpe and J. Singleton Robert, Phys. Rev. D 58, 074501 (1998).

[9] G. Rupak and N. Shoresh, Phys. Rev. D 66, 054503 (2002).

[10] W.-J. Lee and S.R. Sharpe, Phys. Rev. D 60, 114503 (1999).

[11] C. Aubin et al., hep-lat/0209066.

[12] K.G. Wilson, Phys. Rev. D 10, 2445 (1974).

[13] P.H. Ginsparg and K.G. Wilson, Phys. Rev. D 25, 2649 (1982).

[14] P. Hasenfratz, V. Laliena, and F. Niedermayer, Phys. Lett. B 427, 125 (1998).

[15] H. Neuberger, Phys. Lett. B 417, 141 (1998).

[16] H. Neuberger, Phys. Lett. B 427, 353 (1998).

[17] F. Niedermayer, Nucl. Phys. B (Proc. Suppl.) 73, 105 (1999).

[18] M. Luscher, Phys. Lett. B 428, 342 (1998).
[19] P.H. Damgaard, J.C. Osborn, D. Toublan, and J.J.M. Verbaarschot, Nucl. Phys. B547, 305 (1999).

[20] S.R. Sharpe and N. Shoresh, Phys. Rev. D 64, 114510 (2001).

[21] K. Symanzik, in Recent Developments in Gauge Theories, Proceedings, NATO Advanced Study Institute, Cargese, France, 1979, edited by G. 't Hooft et al. (NATO Advanced Study Institute Series, Series B, Physics, Vol. 59) (Plenum, New York, 1980), p. 438.

[22] K. Symanzik, Nucl. Phys. B226, 187 (1983).

[23] K. Symanzik, Nucl. Phys. B226, 205 (1983).

[24] P. Hernández, K. Jansen, and M. Lüscher, Nucl. Phys. B552, 363 (1999).

[25] B. Sheikholeslami and R. Wohlert, Nucl. Phys. B259, 572 (1985).

[26] M. Lüscher, S. Sint, R. Sommer, and P. Weisz, Nucl. Phys. B478, 365 (1996).

[27] S. Weinberg, Physica A 96, 327 (1979).

[28] S.R. Sharpe, Phys. Rev. D 56, 7052 (1997); 62, 099901(E) (2000).

[29] M.F.L. Golterman and K.-C. Leung, Phys. Rev. D 57, 5703 (1998).

[30] P. Hasenfratz, Nucl. Phys. B525, 401 (1998).

[31] P. Hasenfratz, S. Hauswirth, T. Jorg, F. Niedermayer, and K. Holland, Nucl. Phys. B643, 280 (2002). 